\title{
Analysis of crystallographic structures and DFT calculations reveal a new structural arrangement in proteins involving lysine $\mathrm{NH}_{3}{ }^{+}$group and carbonyl.
}

Nikolai R. Skrynnikov, Purdue University, West Lafayette, IN

Sergei A. Izmailov, St. Petersburg State University, St. Petersburg, Russia

Lyudmila V. Slipchenko, Purdue University, West Lafayette, IN

Olga N. Rogacheva, St. Petersburg State University, St. Petersburg, Russia

Lysine side-chain $\mathrm{NH}_{3}{ }^{+}$group is capable of forming three conventional hydrogen bonds. This arrangement creates a "sweet spot" for the fourth electron-rich ligand, such as carbonyl oxygen. Specifically, carbonyl $\mathrm{O}$ atom often occupies the position on the (three-fold) symmetry axis of the $\mathrm{NH}_{3}{ }^{+}$group at a distance 2.7-3.0 $\AA$ from the nitrogen atom. The analysis of high-to-mediumresolution protein structures in the PDB finds more than 10,000 such occurrences, corresponding to a distinct cluster on the phase-space probability density map. Our DFT calculations confirm that this arrangement, which is termed "linear $\mathrm{NH}_{3}{ }^{+}$-carbonyl interaction", offers a stabilizing energy of up to $2 \mathrm{kcal} / \mathrm{mol}$ that is mainly of electrostatic origin. Among many biologically relevant examples, linear interaction involving conserved lysine is consistently found in the P-loop of numerous NTPase domains (including Ras and Ras-like proteins), where it stabilizes the substratebinding conformation of the P-loop. In addition to carbonyl, oxygen atoms of crystallographic water molecules as well as oxygen atoms from Ser, Thr and Tyr side-chain hydroxyl groups can also form a similar linear arrangement with $\varepsilon$-ammonium ion. Curiously, carboxylic oxygens do not engage in linear interactions. It appears that $\mathrm{COO}^{-}$can usually force its way into a more favorable hydrogen-bonded position by displacing a weaker acceptor, such as carbonyl. As a result, $\mathrm{COO}^{-} \cdots \mathrm{NH}_{3}{ }^{+}$salt bridges almost invariably occur in a form of hydrogen bonds.

The research was supported by RSF grant 15-14-20038. 